\title{
Asymmetry of Sunspot Distribution in KAU Solar Observations during 22 and 23 Solar Cycles
}

\author{
Hassan Mohammad Basurah, Amerah Fouad Dhignah, Fouad Yousef Kamal \\ Department of Astronomy, Faculty of Science, King Abudlaziz University, Jeddah, KSA \\ Email: hbasurah@kau.edu.sa \\ Received October 14, 2013; revised November 10, 2013; accepted November 17, 2013
}

Copyright (C) 2013 Hassan Mohammad Basurah et al. This is an open access article distributed under the Creative Commons Attribution License, which permits unrestricted use, distribution, and reproduction in any medium, provided the original work is properly cited.

\begin{abstract}
The King Abdul Aziz University (KAU) solar observations of Sunspot on daily passes from 1981 to 2009 are used to investigate the North-South asymmetry variation of the sunspot numbers and its area. We found that the asymmetry dominated to be southern during the last two solar cycles, which evidently exists following a long-term characteristic time scale. Our results were in compliance with the global asymmetry.
\end{abstract}

Keywords: Sunspots; Long Solar Cycles; North-south Solar Asymmetry

\section{Introduction}

There are many solar activity indices for the solar disk, however all are not identical in the northern or the southern solar hemispheres [1-4]. This North-South asymmetry (ANS) is considered to be a new index to assist in tracing the nature of solar activity and to understand the solar cycles and other phenomena. The asymmetry of sunspot distribution and sunspot area in the two solar hemispheres has been noted since the beginning of the last century by $[5,6]$, and has been further demonstrated by [7-9]. Other solar activity indices such as the number of flares and its index also display similar asymmetry, [10-17]. It is considered that the north-south asymmetry of sunspots is due to periodical activities between the northern and southern hemispheres. The solar asymmetry also shows a number of short and midterm periodicities of the north-south asymmetry of the sunspot area, [7, 18-20]. While a significant long periods have been pointed by $[3,8,21-25]$. Also, it is found to be changing every eight solar cycles according to [10], while [26] found longer time scale of about 12 solar cycles. The asymmetry of the solar activity during the cycles 19 and 20 was northern dominated, while during cycle 21 it was southern dominated, and this characteristic continued during cycles 22 and 23 . We expect that the domination will shift to the north hemisphere by cycle 25 . In this paper a statistical analysis for the north-south solar asymmetry has been performed using both the sunspot numbers and the sunspot areas for period 1981-2009. In
Section 2, the observational data and the results are described. In Sections 3, the conclusions are given.

\section{Measurement and Results}

For the analysis which follows, we have used the daily sunspot maps from the KAU solar observatory. These observations have been taken using the $15 \mathrm{~cm}$ Coudé refractor telescope with a focal length of $225 \mathrm{~cm}$ [27,28].

The twenty eight years of observations, cover the last half of 21, 22 and 23 solar cycles, have been analyzed for both northern and southern sunspots, and smoothed for 13 months as standard way [29]. Figures 1(a) and (b) illustrate the distribution of the sunspot numbers and sunspot areas at both solar hemispheres respectively. To illustrate the asymmetry Equation (1) was used.

$$
\mathrm{ANS}=(\mathrm{Nn}-\mathrm{Ns}) /(\mathrm{Nn}+\mathrm{Ns})
$$

where $\mathrm{Nn}$ and Ns are the active parameters of north $(\mathrm{N})$ or south $(\mathrm{S})$.

Figure 2 shows clearly that the ANS for monthly sunspot areas during solar cycles $22 \& 23$ is southern dominant, as predicted by [30]. Also, the relative sunspot numbers show the same southern trend. A clear relationship is found between the coefficients of north-south asymmetry and the solar cycle numbers 8 to 22 , which tends to be a long-term cycle of approximately of 110 years. Our results fit well with the global results of [31], Figure 3. It is therefore likely that the current solar cycle 24 will be less southern dominant, as the asymmetry of 


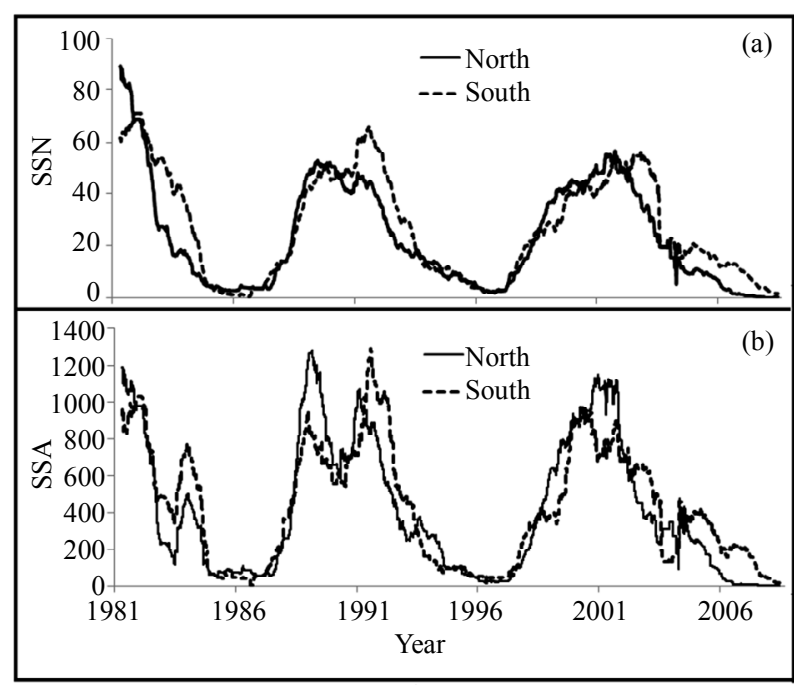

Figure 1. Distribution of the solar sunspot numbers (a) and its areas (b), for the KAU observations, respectively in northern (-) and southern (----) hemispheres.

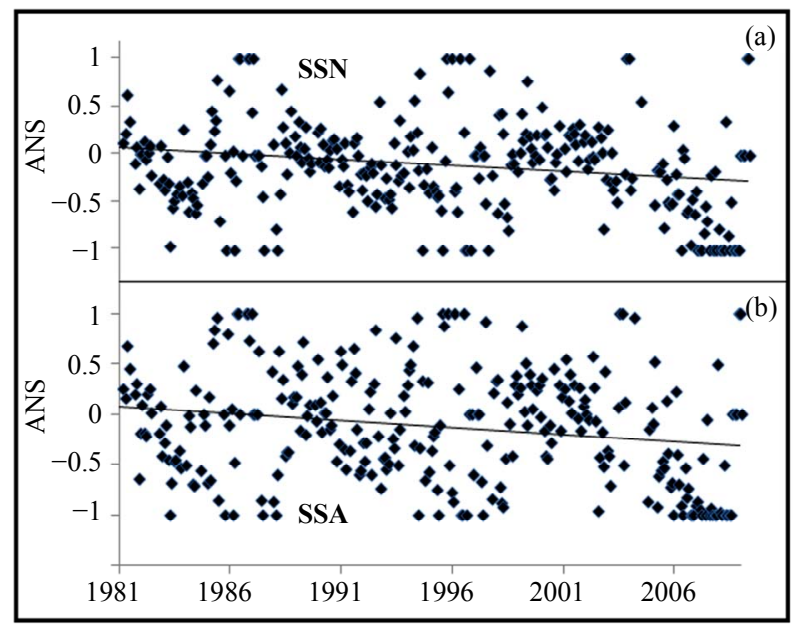

Figure 2. Fit of regression line to mean monthly asymmetry values of the sunspot numbers (above panel) and its areas (lower panel), during the observation time 1981-2009. The negative regression indicate the southern domination.

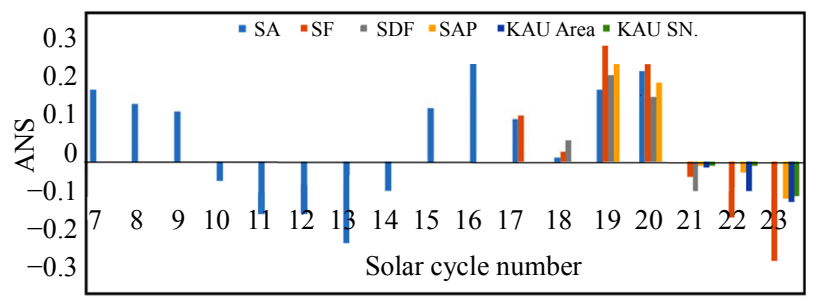

Figure 3. The asymmetry of different solar indices (SA sunspot area, SF solar flare, SDF sudden disappeared filaments, SAP solar active prominence) for previous 17 solar cycles [11]. Our results of the last 3 cycles for the sunspot numbers (KAU SN) and its areas (KAU SA) are fitted well.

the cycle 25 tends to shift to the northern hemisphere,
$[1,20,25]$.

\section{Conclusion}

In this work, we use a new index which describes the asymmetry of sunspot activity in the northern and southern solar hemispheres using observations of KAU solar Observatory between 1981 and 2009. The results agree with the global predictions of the asymmetry. Specifically, we find that the asymmetry was southern dominated on the cycles 21, 22 and 23, in agreement with [26,30]. Also, our results confirm that the southern dominant asymmetry will continue for the cycle 24 , but it may shift to a northern dominance by cycle 25 . This movement to the northern hemisphere seems to follow a long period of the solar activity [3,10,31]. The understanding of the ANS of the solar activity and its long cycle may help predict the level of the solar activity $[32,33]$, as the long cycles are quite important to the terrestrial weather system, because there is a possible connection to the long solar variability to the terrestrial weather [34].

\section{REFERENCES}

[1] V. K. Verma, "On the North-South Asymmetry of Solar Activity Cycles," Astrophysical Journal, Vol. 403, No. 2, 1993, pp. 797-800. http://dx.doi.org/10.1086/172250

[2] K. J. Li and X. M. Gu, "Does the Southern Dominance of Soar Activity Really Exist in Solar Cycle 21?" Astronomy and Astrophysics, Vol. 353, 2000, pp. 396-398.

[3] G. Vizoso and J. L. Ballester, "The North-South Asymmetry of Sunspots," Astronomy and Astrophysics, Vol. 229, 1990, pp. 540-546.

[4] M. Carbonell, J. Terradas, R. Oliver and J. L. Ballester, "The Statistical Significance of the North-South Asymmetry of Solar Activity Revisited," Astronomy and Astrophysics, Vol. 476, No. 2, 2007, pp. 951-957.

http://dx.doi.org/10.1051/0004-6361:20078004

[5] E. W. Maunder, "Note on the Distribution of Sunspots in Heliographic Latitude, 1874-1902," Monthly Notices of Royal Astronomical Society, Vol. 64, 1904, pp. 747-761.

[6] H. W. Newton and A. S. Milson, "Note on the Observed Differences in Spottedness of the Sun's Northern and Southern Hemispheres," Monthly Notices of Royal Astronomical Society, Vol. 115, 1955, pp. 398-404.

[7] J. L. Ballester, R. Oliver and M. Carbonell, "The Periodic Behavior of the North-South Asymmetry of Sunspot Areas Revisited," Astronomy and Astrophysics, Vol. 431, No. 2, 2005, pp. L5-L8. http://dx.doi.org/10.1051/0004-6361:200400135

[8] R. Oliver and J. L. Ballester, "The North-South Asymmetry of Sunspot Area during Solar Cycle 22," Solar Physics, Vol. 152, No. 2, 1994, pp. 481-485.

[9] S. I. Zharkov and V. V. Zharkova, "Statistical Analysis of the Sunspot Area and Magnetic Flux Variations in 1996 2005 extracted from the Solar Feature Catalogue," Ad- 
vances in Space Research, Vol. 38, No. 5, 2006, pp. 868875. http://dx.doi.org/10.1016/j.asr.2006.03.035

[10] T. Atac and A. Ozguc, "North-South Asymmetry in the Solar Flare Index," Solar Physics, Vol. 166, No. 1, 1996, pp. 201-208.

[11] T. Atac and A. Ozguc, "Flare Index during the Rising Phase of Solar Cycle 23," Solar Physics, Vol. 198, No. 2, 2001, pp. 399-407. http://dx.doi.org/10.1023/A:1005218315298

[12] T. Atac and A. Ozguc, "Overview of the Solar Activity during Cycle 23," Solar Physics, Vol. 233, No. 1, 2006, pp. 139-153.

http://dx.doi.org/10.1007/s11207-006-1112-3

[13] K. J. Li, X. Gu, F. Xiang, X. Liu and X. Chen, "Sunspot Groups at High Latitude," Monthly Notices of Royal Astronomical Society, Vol. 317, No. 4, 2000, pp. 897-901. http://dx.doi.org/10.1046/j.1365-8711.2000.03758.x

[14] K. J. Li, J. X. Wang, L. S. Zhan, H. S. Yun, H. F. Liang, H. J. Zhao and X. M. Gu, "On the Latitudinal Distribution of Sunspot Groups over a Solar Cycle," Solar Physics, Vol. 215, No. 1, 2003, pp. 99-109. http://dx.doi.org/10.1023/A:1024814505979

[15] A. Ozguc and T. Atac, "Effects of Hysteresis in Solar Cycle Variations between Flare Index and Cosmic Rays," New Astronomy, Vol. 8, No. 8, 2003, pp. 745-750. http://dx.doi.org/10.1016/S1384-1076(03)00063-0

[16] A. Ozguc, T. Atac and J. Rybak, "Temporal Variability of the Flare Index (1966-2001)," Solar Physics, Vol. 214, No. 2, 2003, pp. 375-396. http://dx.doi.org/10.1023/A:1024225802080

[17] A. Ozguc, T. Atac and J. Rybak, "Evaluation of the Short-Term Periodicities in the Flare Index between the Years 1966-2002," Solar Physics, Vol. 223, No. 1, 2004, pp. 287-304. http://dx.doi.org/10.1007/s11207-004-7304-9

[18] P. Chowdhury and B. N. Dwivedi, "A Study of the North-South Asymmetry of Sunspot Area during Solar Cycle 23," In: A. R. Choudhuri and D. Banerjee, Eds., First Asia-Pacific Solar Physics Meeting ASI Conference Series, Vol. 2, 2011, pp. 197-201.

[19] P. Chowdhury, D. P. Choudhary and S. Gosain, "A Study of the Hemispheric Asymmetry of Sunspot Area during Solar Cycles 23 and 24," The Astrophysical Journal, Vol. 768, No. 2, 2013, pp. 10-15.

[20] H. Y. Chang, "Variation in North-South Asymmetry of Sun Spot Area," Journal of Astronomy and Space Sciences, Vol. 24, No. 2, 2007, pp. 91-98. http://dx.doi.org/10.5140/JASS.2007.24.2.091

[21] R. Knaack, J. O. Stenflo and S. V. Berdyugina, "Periodic Oscillations in the North-South Asymmetry of the Solar Magnetic Field," Astronomy and Astrophysics, Vol. 418, No. 1, 2004, pp. L1-L4. http://dx.doi.org/10.1051/0004-6361:20040106
[22] R. Oliver and J. L. Ballester, "Rescaled Range Analysis of the Asymmetry of Solar Activity," Solar Physics, Vol. 169, No. 1, 1996, pp. 215-224. http://dx.doi.org/10.1007/BF00153842

[23] P. J. Pulkkinen, J. Brooke, J. Pelt and I. Tuominen, "Long-Term Variation of Sunspot Latitudes," Astronomy and Astrophysics, Vol. 341, 1999, pp. L43-L46.

[24] S. Zharkov, V. V. Zharkova and S. S. Ipson, "Statistical Properties of Sunspots in 1996-2004: I. Detection, NorthSouth Asymmetry and Area Distribution," Solar Physics, Vol. 228, No. 1-2, 2005, pp. 377-397. http://dx.doi.org/10.1007/s11207-005-5005-7

[25] K.J. Li, H. S. Yun and X. M. Gu, "Hemispheric Variation in Solar Activity," The Astrophysical Journal, Vol. 554, No. 1, 2001, pp. L115-L117. http://dx.doi.org/10.1086/320914

[26] K.J. Li, P. X. Gao and L. S. Zhan, "The Long-Term Behavior of the North-South Asymmetry of Sunspot Activity," Solar Physics, Vol. 254, No. 1, 2009, pp. 145-154. http://dx.doi.org/10.1007/s11207-008-9284-7

[27] H. M. Basurah, Y. M. Almleaky and A. A. Malawi, "The Solar Activity Maximum of Cycle No. 22 from KAAU Solar Observatory," Astrophysics and Space Science, Vol. 215, No. 1, 1994, pp. 131-133.

[28] H. M. Basurah, "The K.A.A.U. Solar Observatory," IAPPP. Communication, Vol. 62, 1996, pp. 1-4.

[29] T. Podladchikova, B. Lefebvre and R. van der Linden, "Integral Activity of the Declining Phase of Sunspot Cycles as Precursor of the Next Cycle," Journal of Atmospheric and Solar-Terrestrial Physics. Vol. 70, No. 2-4, 2008, pp. 277-287. http://dx.doi.org/10.1016/j.jastp.2007.08.068

[30] V. K. Verma, "The Distribution of the North-South Asymmetry for the Various Activity Cycles," ASP Conference Series, Vol. 27, 1992, pp. 429-435.

[31] V. K. Verma, "Periodic Variation of the North-South Asymmetry of Solar Activity Phenomena," Journal of Astrophysics and Astronomy, Vol. 21, No. 3-4, 2000, pp. 173-176. http://dx.doi.org/10.1007/BF02702386

[32] D. H. Hathaway and R. M. Wilson, "What the Sunspot Record Tells Us about Space Climate," Solar Physics, Vol. 224, No. 1-2, 2004, pp. 5-19. http://dx.doi.org/10.1007/s11207-005-3996-8

[33] J. Javaraiah, "Sun's Retrograde Motion and Violation of Even-Odd Cycle Rule in Sunspot Activity," Monthly Notices of Royal Astronomical Society, Vol. 362, No. 4, 2005, pp. 1311-1318. http://dx.doi.org/10.1111/j.1365-2966.2005.09403.x

[34] H. M. Basurah, "Nile Flooding Fluctuations and Its Possible Connection to the Long Solar Variability," Journal of the Association of Arab Universities for Basic and Applied Sciences, Vol. 1, 2005, pp. 1-7. 